Voix et Images

volxetimages

\title{
Journaux fictifs / fiction diaristique
}

Jean-Louis Major

Volume 20, numéro 1 (58), automne 1994

Saint-Denys Garneau

URI : https://id.erudit.org/iderudit/201149ar

DOI : https://doi.org/10.7202/201149ar

Aller au sommaire du numéro

Éditeur(s)

Université du Québec à Montréal

ISSN

0318-9201 (imprimé)

1705-933X (numérique)

Découvrir la revue

Citer cet article

Major, J.-L. (1994). Journaux fictifs / fiction diaristique. Voix et Images, 20(1),

200-205. https://doi.org/10.7202/201149ar d'utilisation que vous pouvez consulter en ligne.

https://apropos.erudit.org/fr/usagers/politique-dutilisation/ 


\section{Journaux fictifs / fiction diaristique}

Jean-Louis Major, Université d'Ottawa

Tenir son journal exige justification. Tel est le présupposé indiscuté d'ouvrages comme ceux d'Alain Girard ${ }^{1}$ et de Béatrice Didier ${ }^{2}$, qui empruntent volontiers leurs explications à la psychologie. Malgré son titre ${ }^{3}$ et ses diatribes, celui, plus récent, de Georges Gusdorf n'y change pas grand-chose en prenant appui sur une philosophie traditionnelle du sujet. Jean Rousset ${ }^{4}$ et Pierre Hébert ${ }^{5}$ proposent au contraire une lecture qui reconnait dans les journaux intimes un fonctionnement textuel ou, à tout le moins, une structure narrative.

Distinctly Narcissistic : Diary Fiction in Quebec ${ }^{6}$ joint; en principe, l'approche textuelle à l'explication psychólogique: Valerie Raoul y propose une étude de la fiction diaristique, c'est-à-dire des journaux intimes dans le roman québécois. Le genre a une ampleur assez étonnante. Y figurent les romans qui adoptent à des degrés divers la forme du journal, de même que ceux qui donnent des extraits de journaux intimes ou y font allusion. D'Un amour vrai (1878) de Laure Conan à Babel: prise deux (1990) de Francine Noël, le corpus comprend des œuvres essentielles aussi bien que des romans dont on peut dire qu'ils ne sont pas souvent relus. 
Comme la plupart des observateurs extérieurs, Valerie Raoul donne à son étude des romans québécois un cadre sociologique. Mais son approche ne se démarque pas radicalement de celle qui, à un titre ou l'autre, prévaut au Québec. À une différence près. Valerie Raoul se propose d'expliquer l'évolution de la société québécoise à un public intéressé et sympathique (ou inquiet), alors que la plupart des critiques québécois se soucient moins d'expliquer la société que d'affirmer sa particularité.

Dans Distinctly Narcissistic, le cadre sociologique se double d'une perspective psychologique, sous l'égide du narcissisme. La référence est cependant assez polyvalente pour rendre compte de tous les cas de journaux fictifs, du xIX ${ }^{e}$ siècle à aujourd'hui. Le narcissisme peut se traduire par l'attachement à soi, la peur de la castration, le désir de fusion avec l'autre ou l'inscription dans l'ordre symbolique, aussi bien que par un désir de retour au sein maternel, un attachement à la mère pré-œdipienne, la nostalgie de l'éden perdu ou l'inscription dans l'ordre imaginaire. Il se manifeste par l'admiration de soi et la mégalomanie ou, à l'inverse, par l'impuissance et la passivité; par un manque de sensibilité à l'égard d'autrui ou par une identification excessive à l'autre; par la crainte de l'assimilation ou par la quête d'identité: Le narcissisme peut affecter les rapports quotidiens dans leurs formes les plus concrètes ou être sublimé dans l'ordre moral. Selon certains, il est normal et nécessaire; selon les autres, il constitue un état pathologique; les uns y voient un stade du développement; d'autres, un aspect permanent de la personnalité. Chose certaine, il comporte assez d'aspects et de valeurs contradictoires pour servir de référence à des œuvres aussi diverses qu'Angéline de Montbrun et Salut Galarneau!, Les Abîmes de l'aube et Le Libraire, Ce qu'il faut de regrets et Trou de mémoire. Mais surtout, il est le schéma explicatif qui vient à l'esprit dès qu'il s'agit du journal intime. On évoque alors le retrait narcissique, l'isolement, le "refuge autistique ", le rôle tout ensemble de sujet et d'objet que remplit le scripteur, le sens de la discontinuité temporelle, la nostalgie du passé, la peur de la fragmentation, la recherche d'unité, la volonté de contrôle par l'exercice de la lucidité, le désir de renaître par l'écriture, le paradoxe d'écrire pour soi.

Distinctly Narcissistic regroupe les romans selon leurs affinités, mais en les inscrivant dans un cadre chronologique. Les journaux fictifs sont alors lus comme exemplaires d'une étape de l'évolution culturelle ou, plus précisément, du narcissisme collectif. Dans cette perspective, les rapports des diaristes avec les femmes et l'expérience subjective de ces dernières représentent un axe privilégié, en même temps que le lien le plus effectif entre le particulier et le collectif. 
Valerie Raoul s'attache aux romans dont la forme narrative se rapproche le plus du journal intime, mais en donnant au genre un sens large. Dans la plupart des cas, par exemple, les journaux fictifs ne: portent pas de dates, alors que la datation des entrées est l'une des marques les plus explicites du journal intime. Elle prend pour point de départ le Journal d'Henriette Dessaulles, qu'elle rapproche de l'œuvre de Laure Conan. Ainsi, le Journal d'Henriette Dessaulles illustrerait la construction d'une féminité qui prescrit l'abnégation, en faveur du mariage et de la maternité, alors qu'Angéline de Montbrun refléterait le narcissisme moral et la féminisation de la culture qui donnera lieu aux figures de la mère phallique.

Si les journaux fictifs sont à peu près inexistants au début du vingtième siècle, ceux qui apparaissent dans les années quarante et cinquante confirment la faiblesse des figures masculines, voire leur impuissance ou leur castration. Les narrateurs. du Journal d'Anatole Laplante, des. Médisances de Claude Perrin et de Mon fils pourtant beureux se perçoivent comme intellectuels français et, partant, comme efféminés. Les romans en forme de journal de Françoise Loranger, de Robert Élie et de Jean Filiatrault décrivent des narrateurs masculins dont l'impuissance, mise au compte de la domination féminine, les conduit à la folie. Les mères phalliques produisant des fils homosexuels, l'introjection féminine par des narrateurs masculins se manifeste, au cours des années soixante, dans des journaux fictifs dont la thématique est l'homosexualité, d'ailleurs latente ou implicite dans les cuvres antérieures. Ce sont, par exemple, Les Abîmes de l'aube de Jean-Paul Pinsonneault et L'Insoumise de Marie-Claire Blais, symptomatiques du narcissisme adolescent qui s'identifie à la mère, alors que le Journal d'un bobo de Jean-Jules Richard amplifie l'ambivalence sexuelle dans les errances d'un narrateur hermaphrodite. Dans les années quatre-vingt, Michel Tremblay (Des nouvelles d'Édouard) et Madeleine Monette (Le Double suspect) renouvelleront ces thèmes en utilisant aussi des formes du journal fictif.

Les narrateurs du Libraire et de Salut Galarneau! exprimeraient un désir de s'identifier à la mère phallique en donnant naissance à un livre et à soi-même. Une image analogue sous-tendrait le roman de Geneviève Amyot, Journal de l'année passée, mais avec dès significations autres, du fait que l'auteur du journal y est une femme. D'autre part, dans les romans de Paule Saint-Onge ( $\mathrm{Ce}$ qu'il faut de regrets et La Saison de l'inconfort), de Yolande Chéné (Peur et amour) et de Claude Lamarche (Je me veux), les narratrices chercheraient à se libérer (de l'homme) par une forme d'écriture. Ce thème du passage de la spécularité narcissique à la projection d'une voix, au moyen du jour- 
nal intime, sera repris en 1990, mais dans une forme plus complexe, par Michèle Mailhot dans Le Passé composé, pour illustrer le caractère problématique du parallèle entre la situation de la femme et celle de l'homme dans le contexte québécois. Chez Ducharme (Le Nez qui voque) et Aquin (Trou de mémoire), la forme du journal marquerait une volonté de subvertir l'ordre patriarcal qui régit le langage et les rapports entre les sexes. Quant au roman de Francine Noël, Babel: prise deux, il illustrerait la multiplicité de l'autre dans un Québec multiethnique et les dangers du retrait narcissique.

Deux journaux réels, celui d'Henriette Dessaulles et celui de Nicole Brossard, encadrent cette étude de la fiction diaristique. On pourrait soutenir que c'est une façon de définir le paradigme du genre étudié dans les romans, mais ces deux textes sont précisément ceux qui suscitent les questions les plus radicales à l'égard de la forme mêmè du journal intime: l'un, par son agencement postérieur à la rédaction; l'autre (Journal ou Voilà donc un manuscrit, de Nicole Brossard), commandé pour la radio, par les circonstances de sa rédaction et de sa publication, mais plus encore par la problématique des rapports avec la fiction. L'agencement, toutefois, traduit assez bien une approche selon laquelle il n'y a pas de différence essentielle entre personnage de roman et personne réelle. En fin de compte, pourquoi des journaux fictifs? Ce n'est pas l'approche qui détermine le corpus. Qu'y a-t-il de spécifiquement narcissique dans le journal fictif, par opposition à l'autobiogrảphie ou au roman à la première personné ou même à la troisième personne? À chacun des repères qui jalonnent l'ouvrage de Valerie Raoul, on pourrait tout aussi bien rattacher les autres romans de la même période qui n'ont aucun rapport avec le' journal intime.

Le journal fictif correspond à une forme extrinsèque qui intervient dans le roman, avec des caractéristiques, des valeurs et un fonctionnement qui lui sont propres. Le corpus établi par Valerie Raoul appelle une analyse du mode d'inscription'du journal intime dans les romans. $\mathrm{Au}$ lieu de le traiter comme un sous-genre (la "fiction diaristique"), on l'étudierait comme un élément de la forme romanesque. À ce titre, on devrait le considérer de la même façon que les autres formes extrinsèques dans le roman, telles que la correspondance, la publicité, la poésie, les documents, les ouvrages savants ou les chansons. En somme, comme toute forme textuelle qui, dans le roman, suscite une lecture particulière. Ce serait poser les conditions d'une véritable intertextualité des formes.

D'un point de vue formel, rien ne permet de distinguer le journal fictif du journal réel ou le roman à la première personne de la narration 
autobiographique non fictive ${ }^{7}$. Le journal fictif se présente comme une espèce particulière de narration à la première personne. En revanche, en tant que forme extrinsèque, le journal connote des valeurs qui sont mises en œuvre dès qu'on l'inscrit de façon explicite dans le roman. Par exemple, dans le roman de Yolande Chéné: “je méprise ceux qui tiennent un journal". Ou encore, selon Valerie Raoul, au $\mathrm{xIx}^{\mathrm{e}}$ siècle on considérait que tenir un journal n'exige ni intelligence, ni éducation, ni maîtrise du style. Selon Yolande Villemaire, le journal intime est "une cartographie insensée de l'insignifiant". On notera d'ailleurs qu'on n'écrit pas son journal: on le tient. C'est que le journal intime est étranger à la littérature ou qu'il en est exclu: il ne saurait donc donner lieu à une lecture à l'égal du roman lui-même. S'il est lu, c'est comme catalyseur de vérité ou comme voie d'accès à la réalité intérieure.

Dans une perspective "réaliste ", toute narration à la première personne suscite une difficulté, du seul fait qu'elle est écrite. Parce qu'il n'appartient pas à la littérature, le journal permet d'affecter un caractère "naturel "à l'écriture: il en fournit une justification plausible. C'est sans doute ce qui explique sa fréquence dans le roman. Mais, dans la plupart des cas, il n'en reste pas moins une forme particulière de narration: une écriture à proximité des événements, par opposition au récit qui bénéficie d'une vue d'ensemble. Forme ouverte, fragmentaire, toujours en devenir, le journal s'oppose à l'œuvre, forme fermée, logique, complète. C'est précisément en se fondant sur cette opposition que Barthes récusait le journal ${ }^{8}$, alors que les valeurs mises en jeu par cette opposition auraient dû le conduire à la conclusion inverse, plus conforme à la logique de sa propre démarche. Comme quoi les valeurs que connote le journal intime peuvent obnubiler même les esprits les plus éclairés. Imaginons ce qu'il en est des personnages de roman.

Ce sont des perspectives que suggère, par défaut en quelque sorte, une lecture de l'ouvrage de Valerie Raoul. Mais elles prennent leur point de départ dans le corpus qu'elle étudie, en fonction d'autres préoccupations.

1: Alain Girard, Le Journal intime, Paris, Presses universitaires de France, 1963.

2. Béatrice Didier, Le Journal intime, Paris, Presses universitaires de France, 1976.

3. Georges Gusdorf, Les Écritures du moi, premier de deux tomes de Lignes de vie, Paris, Odile Jacob, 1991.

4. Jean Rousset, Le Lecteur intime, Paris, Corti, 1986.

5. Pierre Hébert, Le Journal intime au Québec, Montréal, Fides, 1988.

6. Valerie Raoul, Distinctly Narcissistic: Diary Fiction in Quebec, Toronto, University of Toronto Press, 1993, 307 p. 
7. Kate Hamburger, Logique des genres littéraires, Paris, Seuil, 1986.

8. Voir Roland Barthes, "Une leçon de sincérité ", Poétique, n 47, septembre 1981, p. 259-267. 